\title{
The effect of Guizhi decoction on inflammatory response induced by myocardial ischemia
}

\author{
Suhua Zhang ${ }^{1 *}$, Li Han ${ }^{2 *}$, Yingying Wang ${ }^{3}$, Guijing $\mathrm{Liu}^{4}, \mathrm{Haifa} \mathrm{Shi}^{5^{*}}$ (D)
}

\begin{abstract}
We aim to explore the influence of Guizhi decoction(GD) on inflammatory response in myocardial ischemia models. 45 New Zealand rabbits were divided into three groups randomly: sham control group, myocardial ischemia group and myocardial ischemia + Guizhi decoction treatment group. The rabbits were treated with Guizhi decoction or saline for 20 days. After that, the myocardial ischemia model was created by left anterior descending coronary artery ligation. Color Doppler Echocardiography computed the ejection fraction (EF). Serum content of MyD88 and TNF- $\alpha$ and concentration of cardiac markers (CK-MB, $\mathrm{CK}, \mathrm{LDH}$ and $\mathrm{CTnT}$ ) was measured. After ligation, ST segment was elevated. However, 15 rabbits died due to operation and postoperative care, and were excluded from this study. The final sample size was sham control group ( $\mathrm{n}=10)$, myocardial ischemia group $(n=10)$ and Guizhi decoction group $(n=10)$ Compared with the sham control group, the ejection fraction was decreased and the expression of the TLR4, MyD88, TNF- $a, C K-M B, C K, L D H$ and cTnT in myocardial ischemia group was increased $(\mathrm{p}<0.05)$. After treatment with Guizhi decoction, EF was improved and related inflammatory response factors were decreased $(\mathrm{p}<0.05)$. Pretreatment with Guizhi decoction attenuated the level of proinflammatory markers and improved heart function in animal model for myocardial ischemia.
\end{abstract}

Keywords: myocardial ischemia; inflammatory response; Guizhi decoction; echocardiography.

Practical Application: Acute myocardial ischemia is the leading cause of death in most nations. Our results show that pretreatment with Guizhi decoction attenuates the level of proinflammatory markers and improves heart function in animal model for myocardial ischemia. Guizhi decoction may be a protential drug for acute myocardial ischemia.

\section{Introduction}

Acute myocardial ischemia is the leading cause of death in most nations. Even if the patients survive, the myocardial injury influences the life quality and 5-year survival rate. So the disease brings serious threat to human life and health. (Liu et al., 2013) Rapid diagnosis and timely treatment can effectively limit the myocardial infarct size, decrease the myocardial cell death and influence the prognosis of these patients. At present, the early treatment is based on restoration of blood flow, such as interventional therapy and nitrates. However, ischemia and reperfusion after restoration of blood flow will result in inflammatory response and induce cardiomyocyte apoptosis, which will influence the myocardial remodeling. (Eltzschig \& Eckle, 2011)

In fact, the myocardial ischemia was recorded in traditional Chinese medicine. They believed that the disease belonged to the "chest pain" or "heartache" category, which mainly showed blood stasis, qi deficiency, phlegm-turbidness, cold coagulation. (Zhou \& Wang, 2014) Various traditional Chinese medicines were tested to treat the myocardial ischemia. Palmatine, extracted from the coptidis rhizome and forsythoside B, extracted from lamiophlomis rotate can improve myocardial function. (Kim et al., 2009; Jiang et al., 2010) Makhana also has cardioprotective properties through clearing ROS. (Das et al., 2006) Studies showed that some traditional Chinese medicines play important role in cardio-protection through anti- inflammation, such as salvia miltiorrhiza bunge, forsythoside B, schisandra chinensis, ShuMai Decoction and Xiongshao Capsule. (Jiang et al. 2010; Ren et al., 2010; Chiu \& Ko, 2004; Yin et al., 2008; Dong-mei et al., 2013)

Gualou Guizhi decoction (GLGZD), a well-established Traditional Chinese Medicinal formulation, is widely used to treat stroke in China. Previous studies have shown that Guizhi decoction improved neurological function and decreased cerebral ischemic-reperfusion injury through anti-inflammation. (Zhang et al., 2014; Chen et al., 2014) It decreased IL1, TNFa and NF- $\kappa b$, but increased IL10. (Zhang et al., 2015; Hu et al., 2015) Jiao Hong et al.reported that Guizhi Tang inhibited the proinflammatory factors and oxidation in myocardial ischemia and hyperlipidemia rats. (Jiao et al., 2012) These studies suggest that Guizhi decoction play important role in cardioprotection. 
To explore the possible mechanism of cardioprotection, we will examine the change of cardiac function and inflammatory factors, such as TLR4, MyD88, TNF- $\alpha$, CK-MB, CK, LDH and cTnT. The study will be instrumental to provide experimental evidence for the application of Guizhi decoction in myocardial ischemia treatment.

\section{Materials and methods}

\subsection{Reagent and Guizhi decoction}

Pentobarbital Sodium, MyD88 ELISA kit and TNF- $\alpha$ ELISA kit were ordered from OMEGA. CK ELISA kit, CK-MB ELISA kit, LDH ELISA kit and cTnI ELISA kit were all purchased from Ybiotech (Shanghai, China). First strand cDNA synthesis kit and SYBR Green PCR master MIX kit was purchased from Applied Biosystems incorporated (Foster City, CA, USA).

Guizhi decoction $(2 \mathrm{~g} / \mathrm{ml})$ was provided by the affiliated hospital of Hebei engineering university, and was composed of Guizhi 15 g, Radix Paeoniae Alba 15 g, radix glucurrhizae preparata $10 \mathrm{~g}$, salvia miltiorrhiza $15 \mathrm{~g}$, dalbergia odorifera $10 \mathrm{~g}$, immature bitter orange $10 \mathrm{~g}$, allium macrostemon $10 \mathrm{~g}$, ligusticum wallichii $15 \mathrm{~g}$, ginger $15 \mathrm{~g}$ and jujube $15 \mathrm{~g}$. All these compound prescriptions were immersed by $650 \mathrm{ml}$ drinking water in an enamelled ware for $30 \mathrm{~min}$, and then they were decocted by fierce fire. After boiling, the decoction was mildly fired for 20-30 min until $65 \mathrm{ml}$ decoction was left.

\subsection{Animal and groups}

A total of 45 New Zealand rabbits (2.0-2.2 kg) were provided by Laboratory Animal Center of Hebei Medical University. The protocol was approved by the Ethics Committee of Affiliated Hospital of Hebei Engineering University.

They were randomly divided into three groups: sham control group, myocardial ischemia group and Guizhi decoction group. For sham control group, the rabbits' artery was separated, but not ligated. Myocardial ischemia model was established by ligation of the left anterior descending coronary artery of rabbits. In the Guizhi decoction group, rabbits were gavaged via stomach tube with Guizhi decoction in $3 \mathrm{ml} / \mathrm{kg} /$ day dose the day before surgery. Ligation was then performed as the model group. The dose for treatment of myocardial ischemia for rabbits was referred to a study reported by Tian et al., who uncovered the relationship between the dose and the antipyretic effect. (Tian et al., 2013) The rabbits in sham control group and myocardial ischemia groups received equal amount of saline. After operation, rabbits were exposed to heat lamp for maintaining the body temperature, and they were kept in a quite cage on the super clean bench for 20 days. In this study, totally 15 rabbits were excluded from this study. Among them, 4 died because of losing blood during ligation; 6 died after surgery, due to the myocardial infarction $(\mathrm{n}=4)$, congestive heart failure $(\mathrm{n}=1)$, and pneumothorax ( $\mathrm{n}$ $=1$ ); 5 were excluded because of the failure of the modeling (ST segment was not elevated). The final sample size was sham control group, $\mathrm{n}=10$; myocardial ischemia group, $\mathrm{n}=10$; and Guizhi decoction group, $\mathrm{n}=10$.

\subsection{Myocardial ischemia model building}

The rabbits were anesthetized by ear venous administration of $1-1.5 \mathrm{ml} / \mathrm{kg} 3 \%$ pentobarbital sodium. The rabbits were fixed on the operation table when corneal reflection disappeared and muscle tone was decreased. Longitudinal incision near $0.5-1 \mathrm{~cm}$ of left side of sternum was chose. The underlying skin and muscles was separated and 3-4 ribs were cut. Then we cut the pericardium in longitudinal incision. The ligation was operated on 3-5 mm under the upper side of anterior interventricular groove for $20 \mathrm{~min}$. The width and depth of ligation was less than $2 \mathrm{~mm}$ and $1 \mathrm{~mm}$ respectively. Vascular clamp clipped the chest and close the muscles and skins. Electrocardiograph was used to assess if the myocardial ischemia model was successfully established. The whole operation was performed under sterile environment.

\subsection{Cardiac function measuring}

Color Doppler Echocardiography was employed by Toshiba Artida equipment (TOSHIBA, Tokyo, Japan) to assess the cardiac function $4 \mathrm{~h}$ before or after ligation. The rabbits were anesthetized using $10 \%$ chloral hydrate. High frequency probe was used to detect and teichholtz formula was used to calculate the ejection fraction (LV EF\%).

\subsection{ELISA}

Four hours after myocardial ischemia model building, $2 \mathrm{ml}$ blood was harvested and kept in silence for $3 \mathrm{~h}$. The samples were centrifuged at $3000 \mathrm{rpm}$ for $15 \mathrm{~min}$ and the serum were harvested and kept in $-80^{\circ} \mathrm{C}$. Before the ELISA examination, the samples and kit kept in room temperature for $30 \mathrm{~min}$. The standard samples $(100 \mu \mathrm{l})$ and serum samples $(100 \mu \mathrm{l})$ were added into the corresponding well, and then $50 \mu$ lenzyme labeled solution were added into each well. The plate was kept in $37^{\circ} \mathrm{C}$ for $1 \mathrm{~h}$. The plate was washed for 5 times using PBS. $50 \mu \mathrm{l}$ color developing agent $\mathrm{A}$ and $50 \mu \mathrm{l}$ color developing agent $\mathrm{B}$ were added to each well for $15 \mathrm{~min}$ and kept in dark place. Then $50 \mu \mathrm{l}$ stopping solution was added to record the absorbance under $450 \mathrm{~nm}$. According to the standard curve, the concentration of samples was calculated.

\subsection{Real time PCR}

Cardial tissues were isolated and kept in liquid nitrogen. Total RNA was extracted according to the RNA extraction kit (Lexogen, Shanghai, China) and were reversely transcripted into cDNA following First strand cDNA Systhesis Kit (Applied Biosystems incorporated, Foster City, CA, USA). To detect the relative mRNA expression of TLR4, real time PCR was conducted using SYBR Green PCR master MIX kit (AMI). TLR4 Forward primer: GAAAGTATGGTAGGGGTGAAAGCG, TLR4 and Reverse primer: GTGAAGGCAGAGCCGAAA. GAPDH served as internal reference. GAPDH Forward primer: TGGAGTCTACTGGCGTCTT and GAPDH reverse primer: TGTCATATTTCTCGTGGTTCA.

\subsection{Statistical analyses}

The results were expressed as mean $\pm \mathrm{SD}$. One way analysis of variance (ANOVA) by a least-significant-difference test 
was used for statistical comparisons among multiple groups. $\mathrm{p}<0.05$ was considered significant. The data were analyzed using SPSS 19.0 software.

\section{Result}

\subsection{Guizhi decoction improved the heart function of myocardial ischemia rabbits}

Myocardial ischemia model was created by left anterior descending coronary artery ligation (Figure 1). After ligation, the abnormal local movement of ventricular surface was detected with elevated ST segment, suggesting that the myocardial ischemia model was successfully established (Figure 2). Ejection fraction (EF) computed by the results from M-mode and B-mode showed that there was no obvious difference among the three groups before the ligation $(p>0.05)$. Four hours after ligation, the EF was decreased in model group compared with sham control group. After treatment with Guizhi compound, the EF was increased ( $p<0.05)$, indicating that Guizhi compound improved the cardiac function (Figure 3 and Table 1).

\subsection{Guizhi compound prescription decreased the myocardial damage and the inflammatory response induced by myocardial ischemia}

In order to observe the myocardial damage and inflammatory change in myocardial ischemia, we examined the serum content of MyD88, TNF- $\alpha$, CK-MB, CK, LDH and cTnT. We found that compared with the sham control group, the content of the TLR4, MyD88, TNF- $\alpha, \mathrm{CK}-\mathrm{MB}, \mathrm{CK}, \mathrm{LDH}$ and $c \mathrm{TnT}$ in myocardial ischemia model group was increased. After treatment with Guizhi decoction, these indicators were lower in Guizhi decoction group than that of myocardial ischemia model group $(\mathrm{p}<0.05)$ (Table 2). We also examined the TLR4 mRNA expression in cardiac muscle
A

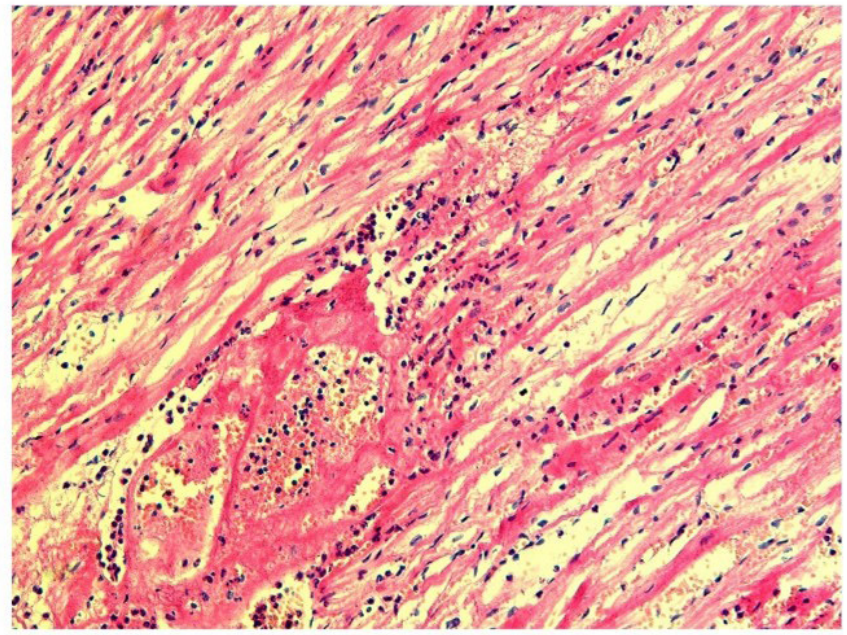

B

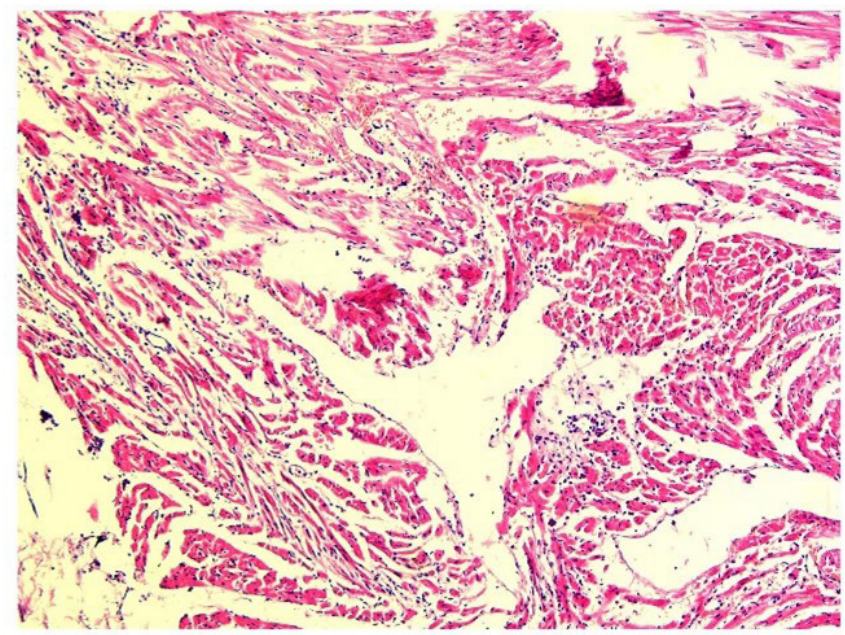

Figure 1. Myocardium tissue of rabbit at $4 \mathrm{~h}$ after surgery $(\times 400)$.

A

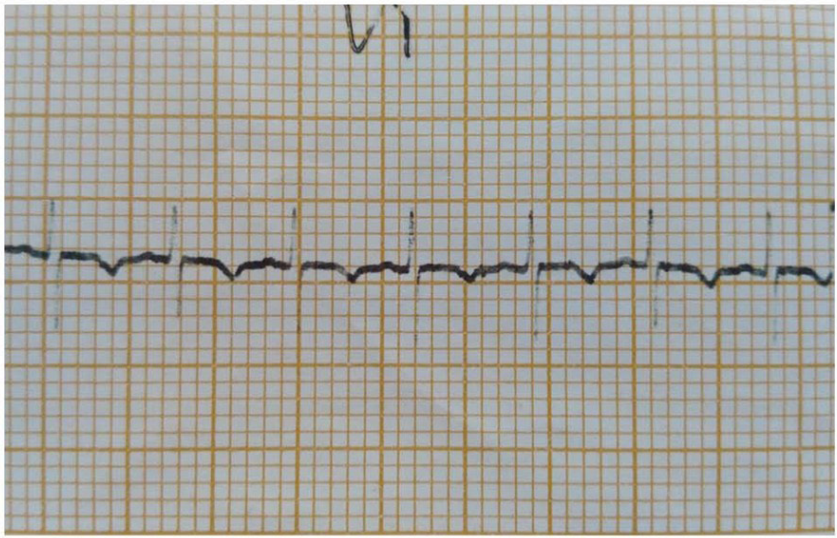

B

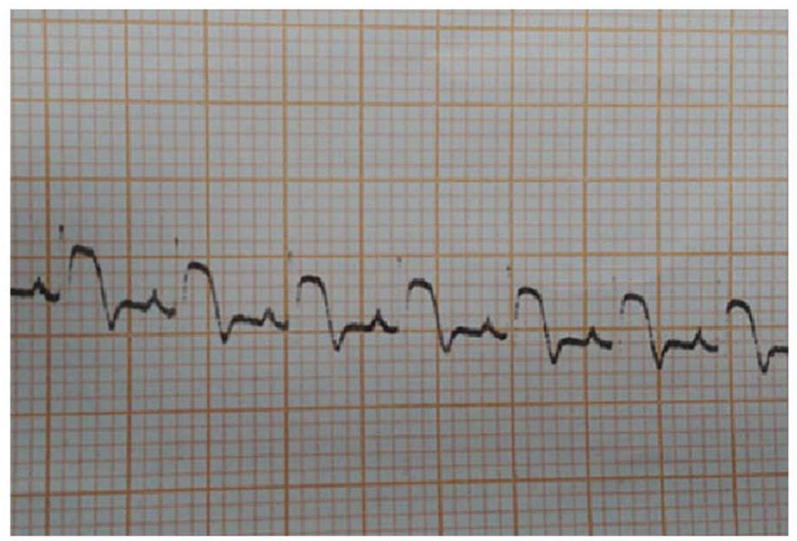

Figure 2. Electrocardiograph. (A) The electrocardiograph before the anterior descending branch of left coronary artery was ligated; (B) The electrocardiograph after the anterior descending branch of left coronary artery was ligated. After ligation, ECG showed that ST segment was elevated. 
A

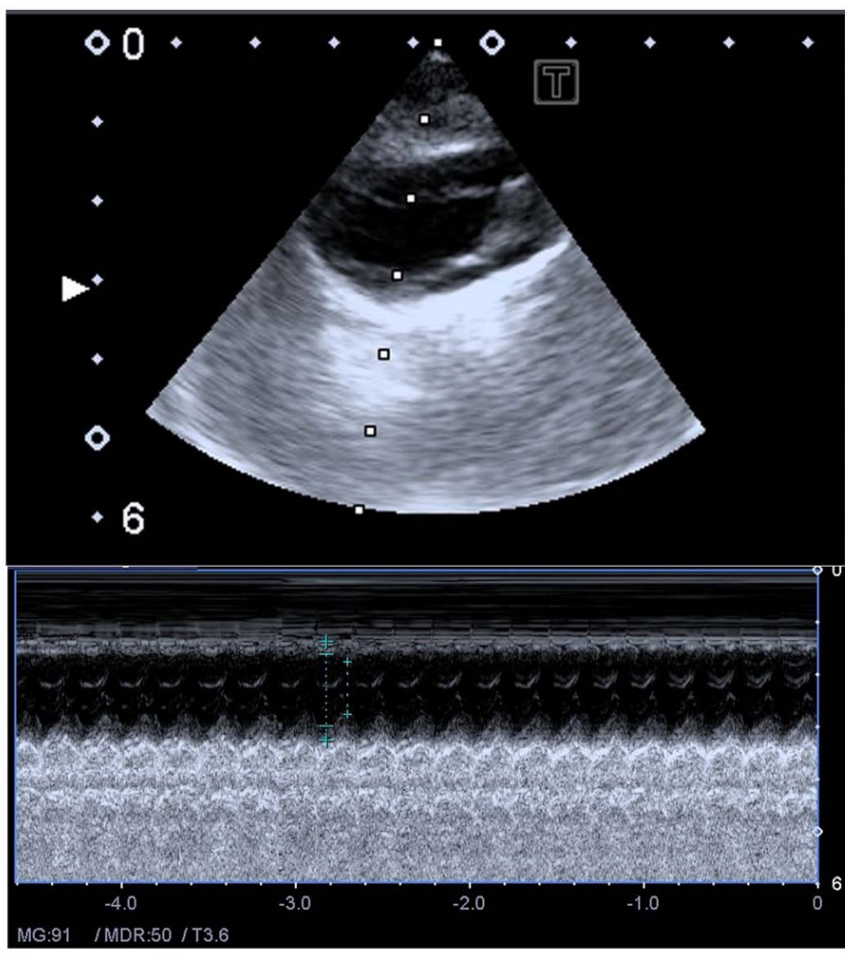

B

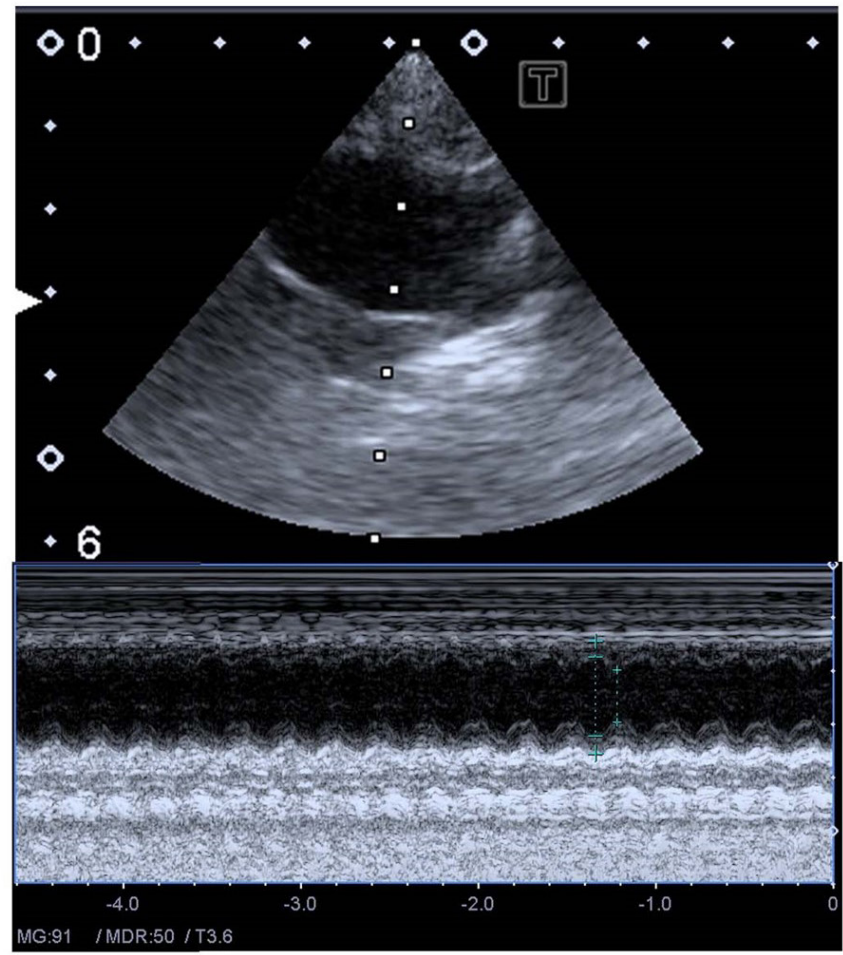

Figure 3. Color Doppler echocardiography. Color Doppler echocardiography was used to assess the ejection fraction. (A) and (B) showed the ejection fraction of myocardial ischemia group and Guizhi decoction group respectively.

Table 1. Ejection fraction (Mean \pm SD).

\begin{tabular}{lccc}
\hline & $\begin{array}{c}\text { Control group } \\
(\mathrm{n}=10)\end{array}$ & $\begin{array}{c}\text { Myocardial } \\
\text { ischemia model } \\
(\mathrm{n}=10)\end{array}$ & $\begin{array}{c}\text { Guizhi } \\
\text { decoction } \\
(\mathrm{n}=10)\end{array}$ \\
\hline $\begin{array}{l}\text { EF before } \\
\text { ligation }\end{array}$ & $71.68 \pm 3.14$ & $72.12 \pm 2.12$ & $71.56 \pm 2.57$ \\
$\begin{array}{l}\text { EF after } \\
\text { ligation }\end{array}$ & $69.32 \pm 2.99$ & $43.57 \pm 3.39^{*}$ & $58.62 \pm 3.54^{\Delta}$ \\
\hline
\end{tabular}

$\cdot P<0.05$ VS control group; ${ }^{\triangle} P<0.05$ VS Myocardial ischemia model.

Table 2. Serum content of MyD88,TNF- $\alpha, C K-M B, C K, L D H$ and cTnT (Mean \pm SD).

\begin{tabular}{lccc}
\hline & $\begin{array}{c}\text { Control group } \\
(\mathrm{n}=10)\end{array}$ & $\begin{array}{c}\text { Myocardial } \\
\text { ischemia model } \\
(\mathrm{n}=10)\end{array}$ & $\begin{array}{c}\text { Guizhi } \\
\text { decoction } \\
(\mathrm{n}=10)\end{array}$ \\
\hline MyD88 (pg/ml) & $164.47 \pm 10.64$ & $428.90 \pm 21.01^{*}$ & $267.17 \pm 16.61^{\Delta}$ \\
TNF- $\boldsymbol{\alpha}(\mathbf{p g} / \mathbf{m l})$ & $49.01 \pm 3.01$ & $110.62 \pm 5.09^{*}$ & $70.90 \pm 5.90^{\Delta}$ \\
CK $(\mathbf{u} / \mathbf{l})$ & $272.67 \pm 26.41$ & $4318.00 \pm 326.01^{*}$ & $2241.00 \pm 136.86^{\Delta}$ \\
CK-MB $(\mathbf{u} / \mathbf{l})$ & $66.67 \pm 7.77$ & $313.34 \pm 22.50^{*}$ & $182.67 \pm 14.61^{\Delta}$ \\
LDH $(\mathbf{u} / \mathbf{l})$ & $86.12 \pm 10.54$ & $343.67 \pm 17.04^{*}$ & $170.33 \pm 7.37^{\Delta}$ \\
cTnT $(\boldsymbol{\mu g} / \mathbf{m l})$ & $0.23 \pm 0.09$ & $1.53 \pm 0.10^{*}$ & $0.73 \pm 0.05^{\Delta}$ \\
\hline${ }^{*} P<0.05 \mathrm{VS}$ control group; ${ }^{\Delta} P<0.05 \mathrm{VS}$ myocardial ischemia model group.
\end{tabular}

tissues and found that relative TLR4 mRNA level was increased in myocardial ischemia model. It was decreased after treatment with Guizhi decoction $(\mathrm{p}<0.05)$ (Table 3).
Table 3. The relative mRNA expression of TLR4 in myocardial tissues (Mean \pm SD).

\begin{tabular}{lccc}
\hline $\begin{array}{c}\text { Control group } \\
(\mathrm{n}=10)\end{array}$ & $\begin{array}{c}\text { Myocardial } \\
\text { ischemia model }(\mathrm{n} \\
=10)\end{array}$ & $\begin{array}{c}\text { Guizhi decoction } \\
(\mathrm{n}=10)\end{array}$ \\
\hline TLR4 & $0.22 \pm 0.03$ & $0.97 \pm 0.08^{\star}$ & $0.43 \pm 0.05^{\Delta}$ \\
\hline${ }^{\circ} P<0.05$ control group; ${ }^{\Delta} P<0.05$ VS Myocardial ischemia model.
\end{tabular}

\section{Discussion}

Acute myocardial ischemia has a high death rates and morbidity. Inflammation happens to distinct stages, including atherosclerotic lesion formation, plaque rupture and repair phase. (Klingenberg \& Luscher, 2012) Inflammation plays important role disease progression and exacerbation. Studies showed that ischemia and reperfusion after restoration of blood flow also resulted in inflammatory response and induced cardiomyocyte apoptosis, which influenced the myocardial remodeling. (Eltzschig \& Eckle, 2011) In this study, we found that Guizhi decoction decreased the inflammatory factors, including TLR4, MyD88 and TNF- $\alpha$ in myocardial ischemia rabbits and improved the cardiac function.

Lactate dehydrogenase (LDH) and creatine kinase (CK) release are usually used to evaluate the presence of necrotic cell death. (Han et al., 2014) Creatine kinase MB isoenzyme (CK-MB) and cardiac troponin $\mathrm{T}$ ( $\mathrm{cTnT}$ ) are sensitive markers for mirror myocardial damage and provide reliable prognostic 
information for myocardial ischemia. (Harris et al., 2000; Nageh et al., 2003) We found that LDH, Ck,CK-MB and cTnT were increased and the ejection fraction was decreased in model group. Guizhi decoction increased the ejection fraction and the makers for myocardial damage was decreased, suggesting that Guizhi decoction indeed released the injury of myocardium and has protective role in ischemia.

Previous studies have shown that TNF- $\alpha$, IL-1B, IL- 6 and NO was released during cardiomyocyte ischemia, which will aggravate the myocardial damage. (Li et al., 2014) TLR4MyD88-p38/NF-kB pathway may contribute to the process. In the myocardial injury/ischemia model, several danger signals such as high mobility group box-1 (HMGB1) are released from the necrotic myocardial cells, then they binds to their receptors such as TLR4 and triggers downstream TLR4MyD88-p38/NF-kB pathway. (Zhang et al., 2017) It has also been well documented that the Toll-like receptor 4 (TLR4)/ NF- $\kappa \mathrm{B}$ signaling pathway mediates early inflammatory responses during myocardial ischemia and reperfusion (MI/R). (Li et al. 2014) Anke Osterloh et al.found that blocking of TLR4 pathway decreased the myocardial inflammatory reaction and reduced the myocardial injury. (Cohen-Sfady et al., 2005; Osterloh et al., 2008; Guo et al., 2011) The potential role of TLR4 in regulating the inflammatory response has been well documented. As well, an effective interdiction of TLR4 might be of great importance to inhibit the adverse myocardial effect. Degradation of I $\kappa B$ will lead to the translocation of NF- $\mathrm{KB}$ p 65 from the cytosol to the nucleus. In myocardial ischemia setting, the changes of TLR4/ NF- $\kappa B$ signaling pathway discussed above will result in enlarged infarct area and increased histologic damage. Our study showed that these inflammatory factors, such as TLR4, MyD88 and TNF- $\alpha$, were increased in model group, indicating that acute myocardial ischemia indeed induced inflammatory reaction. Therefore, it is possible that the Guizhi decoction exert protective effect on myocardium from ischemia by suppressing the TLR4MyD88-NF-кB signaling pathway.

There are several limitations in this study that we should be pointed out. Firstly, we did not perform the chromatography analysis to evaluate which ingredient in the guizhi decoction plays key role in inhibition of level of proinflammatory markers. Second, we just try to explain Guizhi decoction have protective effect on myocardium, while we don't analyze its cardiotonic effect and inhibition of necrosis. The additional experiments regarding time-dependent changes will useful in the comprehension of the pathophysiology. Moreover, the analysis in Guizhi decoction treated animals without ischemia (ie, normal animals) is also helpful.

\section{Conclusion}

In conclusion, application of guizhi decoction relieved the myocardial damage during myocardial ischemia. The decrease of inflammatory response may contribute to the process.

\section{Ethical approval}

The protocol was approved by the Ethics Committee of Affiliated Hospital of Hebei Engineering University.

\section{Conflicts of interest}

All authors declare that they have no conflict of interest.

\section{References}

Chen, X., Li, H., Huang, M., Huang, M., Xu, W., Chu, K., Chen, L., \& Zhang, Y. (2014). Effect of Gua Lou Gui Zhi decoction on focal cerebral ischemia-reperfusion injury through regulating the expression of excitatory amino acids and their receptors. Molecular Medicine Reports, 10(1), 248-254. http://dx.doi.org/10.3892/mmr.2014.2189. PMid:24788947.

Chiu, P. Y., \& Ko, K. M. (2004). Schisandrin B protects myocardial ischemia-reperfusion injury partly by inducing Hsp25 and Hsp70 expression in rats. Molecular and Cellular Biochemistry, 266(1-2), 139-144. http://dx.doi.org/10.1023/B:MCBI.0000049151.79238.30. PMid:15646035.

Cohen-Sfady, M., Nussbaum, G., Pevsner-Fischer, M., Mor, F., Carmi, P., Zanin-Zhorov, A., Lider, O., \& Cohen, I. R. (2005). Heat shock protein 60 activates B cells via the TLR4-MyD88 pathway. Journal of Immunology, 175(6), 3594-3602. http://dx.doi.org/10.4049/ jimmunol.175.6.3594. PMid:16148103.

Das, S., Der, P., Raychaudhuri, U., Maulik, N., \& Das, D. K. (2006). The effect of Euryale ferox (Makhana), an herb of aquatic origin, on myocardial ischemic reperfusion injury. Molecular and Cellular Biochemistry, 289(1-2), 55-63. http://dx.doi.org/10.1007/s11010006-9147-1. PMid:16628469.

Dong-mei, X., Jun-hua, Z., Li-yuan, K., Ming-jun, Z., \& Hong-cai, S. (2013). Traditional Chinese medicine for myocardial infarction: an overview. International Journal of Clinical Practice, 67(12), 1254-1260. http://dx.doi.org/10.1111/ijcp.12172. PMid:24246206.

Eltzschig, H. K., \& Eckle, T. (2011). Ischemia and reperfusion--from mechanism to translation. Nature Medicine, 17(11), 1391-1401. http://dx.doi.org/10.1038/nm.2507. PMid:22064429.

Guo, Y. L., Shen, W., \& Du, F. (2011). Effect of picroside II on expressions of TLR4 and NFkappaB in rats with cerebral ischemia reperfusion injury. Zhongguo Zhong Xi Yi Jie He Za Zhi, 31(1), 58-61. PMid:21434346.

Han, J., Wang, D., Yu, B., Wang, Y., Ren, H., Zhang, B., Wang, Y., \& Zheng, Q. (2014). Cardioprotection against ischemia/reperfusion by licochalcone B in isolated rat hearts. Oxidative Medicine and Cellular Longevity, 2014, 134862. http://dx.doi.org/10.1155/2014/134862. PMid:25215172.

Harris, B. M., Nageh, T., Marsden, J. T., Thomas, M. R., \& Sherwood, R. A. (2000). Comparison of cardiac troponin T and I and CK-MB for the detection of minor myocardial damage during interventional cardiac procedures. Annals of Clinical Biochemistry, 37(Pt 6), 764769. http://dx.doi.org/10.1258/0004563001900075. PMid:11085620.

Hu, H. X., Lin, R. H., Zhu, X. Q., Li, Z. F., \& Chen, L. D. (2015). Antiinflammatory effects of Gualou Guizhi decoction in transient focal cerebral ischemic brains. Molecular Medicine Reports, 12(1), 13211327. http://dx.doi.org/10.3892/mmr.2015.3511. PMid:25815521.

Jiang, W. L., Fu, F. H., Xu, B. M., Tian, J. W., Zhu, H. B., \& Jian-Hou, (2010). Cardioprotection with forsythoside B in rat myocardial ischemia-reperfusion injury: relation to inflammation response. Phytomedicine, 17(8-9), 635-639. http://dx.doi.org/10.1016/j. phymed.2009.10.017. PMid:19959348.

Jiao, H., Ma, J., Chen, Y., Ju, D., Xiang, L., \& Wang, S. (2012). Effects of guizhi tang on inflammatory cytokines in myocardial ischemia and hyperlipidemia rats. Zhongguo Zhongyao Zazhi, 37(11), 16341637. PMid:22993997. 
Kim, Y. M., Ha, Y. M., Jin, Y. C., Shi, L. Y., Lee, Y. S., Kim, H. J., Seo, H. G., Choi, J. S., Kim, Y. S., Kang, S. S., Lee, J. H., \& Chang, K. C. (2009). Palmatine from Coptidis rhizoma reduces ischemiareperfusion-mediated acute myocardial injury in the rat. Food and Chemical Toxicology, 47(8), 2097-2102. http://dx.doi.org/10.1016/j. fct.2009.05.031. PMid:19497345.

Klingenberg, R., \& Luscher, T. F. (2012). Inflammation in coronary artery disease and acute myocardial infarction - is the stage set for novel therapies? Current Pharmaceutical Design, 18(28), 4358-4369. http://dx.doi.org/10.2174/138161212802481219. PMid:22390645.

Li, T., Yu, J., Chen, R., Wu, J., Fei, J., Bo, Q., Xue, L., \& Li, D. (2014). Mycophenolate mofetil attenuates myocardial ischemia-reperfusion injury via regulation of the TLR4/NF-kappaB signaling pathway. Die Pharmazie, 69(11), 850-855. PMid:25985583.

Liu, Q., Li, J., Wang, J., Li, J., Janicki, J. S., \& Fan, D. (2013). Effects and mechanisms of chinese herbal medicine in ameliorating myocardial ischemia-reperfusion injury. Evidence-Based Complementary and Alternative Medicine, 2013, 925625. http://dx.doi.org/10.1155/2013/925625. PMid:24288571.

Nageh, T., Sherwood, R. A., Harris, B. M., Byrne, J. A., \& Thomas, M. R. (2003). Cardiac troponin T and I and creatine kinase-MB as markers of myocardial injury and predictors of outcome following percutaneous coronary intervention. International Journal of Cardiology, 92(2-3), 285-293. http://dx.doi.org/10.1016/S01675273(03)00105-0. PMid:14659867.

Osterloh, A., Veit, A., Gessner, A., Fleischer, B., \& Breloer, M. (2008). Hsp60-mediated T cell stimulation is independent of TLR4 and IL-12. International Immunology, 20(3), 433-443. http://dx.doi. org/10.1093/intimm/dxn003. PMid:18256040.

Ren, Z. H., Tong, Y. H., Xu, W., Ma, J., \& Chen, Y. (2010). Tanshinone II A attenuates inflammatory responses of rats with myocardial infarction by reducing MCP-1 expression. Phytomedicine, 17(3-4), 212-218. http://dx.doi.org/10.1016/j.phymed.2009.08.010. PMid:19800776.

Tian, J., Guo, X., Liu, X. M., Liu, L., Weng, Q. F., Dong, S. J., Knowlton, A. A., Yuan, W. J., \& Lin, L. (2013). Extracellular HSP60 induces inflammation through activating and up-regulating TLRs in cardiomyocytes. Cardiovascular Research, 98(3), 391-401. http:// dx.doi.org/10.1093/cvr/cvt047. PMid:23447644.

Yin, H. Q., Wang, B., Zhang, J. D., Lin, H. Q., Qiao, Y., Wang, R., \& Liu, F. Y. (2008). Effect of traditional Chinese medicine Shu-Mai-Tang on attenuating TNFalpha-induced myocardial fibrosis in myocardial ischemia rats. Journal of Ethnopharmacology, 118(1), 133-139. http:// dx.doi.org/10.1016/j.jep.2008.03.022. PMid:18486373.

Zhang, Y., Li, H., Huang, M., Chu, K., Xu, W., Zhang, S., Que, J., \& Chen, L. (2014). Neuroprotective effects of Gualou Guizhi decoction in vivo and in vitro. Journal of Ethnopharmacology, 158(Pt A), 76-84. http://dx.doi.org/10.1016/j.jep.2014.10.020. PMid:25456424.

Zhang, Y., Zhang, S., Li, H., Huang, M., Xu, W., Chu, K., Chen, L., \& Chen, X. (2015). Ameliorative effects of Gualou Guizhi decoction on inflammation in focal cerebral ischemic-reperfusion injury. Molecular Medicine Reports, 12(1), 988-994. http://dx.doi.org/10.3892/ mmr.2015.3515. PMid:25815894.

Zhang, J. J., Peng, K., Zhang, J., Meng, X. W., \& Ji, F. H. (2017). Dexmedetomidine preconditioning may attenuate myocardial ischemia/reperfusion injury by down-regulating the HMGB1-TLR4MyD88-NF-кB signaling pathway. PLoS One, 12(2), е 0172006.

Zhou, W., \& Wang, Y. (2014). A network-based analysis of the types of coronary artery disease from traditional Chinese medicine perspective: potential for therapeutics and drug discovery. Journal of Ethnopharmacology, 151(1), 66-77. http://dx.doi.org/10.1016/j. jep.2013.11.007. PMid:24269247. 